\title{
Automated Measurement of Hepatic Fat in T1- Mapping and DIXON MRI as a Powerful Biomarker of Metabolic Profile and Detection of Hepatic Steatosis
}

\author{
Khouloud AFFI ${ }^{1}$, Mnaouer KACHOUT ${ }^{2}$ \\ College of Science, Gafsa University, Gafsa, Tunisia ${ }^{1}$ \\ Department of Computer Engineering, College of Computer Science and Engineering, University of Hail, KSA ${ }^{2}$ \\ Innov'COM, Sup'Com, Carthage University, Tunis, Tunisia ${ }^{2}$
}

\begin{abstract}
Abnormal or excessive excess of intraperitoneal fat at different anatomical sites (heart, kidneys, liver, etc.) alters the metabolic profile by generating diseases causing cardiovascular complications. These include hepatic steatosis, which requires being increased surveillance before its severe progression to cirrhosis and its complications. Our objective in this study (invivo) was to propose a new approach to characterize and quantify hepatic fat. Then, differentiated patients with metabolic diseases, obesity, Type 2 diabetes (T2D), metabolic syndrome and healthy subjects. This distinction was not only according to traditional measurement tools such as body mass index (BMI) and waist circumference, but also according to the amount of fat from magnetic resonance imaging (MRI) DIXON image and T1mapping at 1.5 Tesla (T). The evaluation results show that our proposed approach is reproducible, fast and robust. The distribution of the amount of hepatic fat in a cohort of data composed of four groups shows that hepatic fat is able to differentiate the metabolic population on the study chest. Relationship study of hepatic fat and cardiovascular parameters shows that hepatic fat is able to differentiate the metabolic population on the study chest. The relationship study of hepatic fat and cardiovascular parameters shows that hepatic fat has a negative influence on the heart if the amount it increases.
\end{abstract}

Keywords-Nonalcoholic fatty liver disease; non-alcoholic steatohepatitis; image processing; metabolic diseases; magnetic resonance imaging; active contour

\section{INTRODUCTION}

Nonalcoholic fatty liver disease (NAFLD) is one of the most common cause of chronic liver disease, its prevalence is rising worldwide and is estimated to affect $30 \%$ of adults and $10 \%$ of children in the United States [1]-[2]. Its rates are rising internationally alongside the growing epidemics of diabetes, obesity, and metabolic syndrome [3]-[4].

NAFLD is encompasses a range of liver histology severity in the absence of chronic alcohol use [5]-[6], it is commonly classified into two phenotypes, non-alcoholic fatty liver (NAFL) and non-alcoholic steatohepatitis (NASH). The most form is simple steatosis in which triglyceride accumulates within hepatocytes. A more advanced form of NAFLD, nonalcoholic steatohepatitis, includes inflammation and liver cell injury. The development of NASH is associated with an in- creased risk for morbidity and mortality through hepatic (fibrosis, cirrhosis, hepatocellular carcinoma) and non-hepatic (cardiovascular disease and cancer) complications [7]-[8].

The underlying cause of NAFLD, insulin resistance, leads to intracellular accumulation of triglycerides in hepatocytes (steatosis) [9]-[10]. Currently, therapeutic trials in NASH require medical imaging techniques that have greatly contributed to the detection of liver steatosis such as biopsy, ultrasound, computed tomography (CT) and recently Magnetic Resonance Imaging (MRI). The gold standard for establishing diagnosis as well as severity of NAFLD is liver biopsy, but it is invasive, poor patient acceptance, requires of a hospitalization, not exempt of complications and suffers from tremendous sampling variability [11].

Diana Feier, Ahmed Ba-Ssalmah and al. estimated that only a tiny fraction of the liver (roughly 1/50.000), leading to sampling errors [12]. They also showed that liver biopsy samples contain at least 11 portable triads and measure at least $2.0 \mathrm{~cm}$ to reduce sampling variability. Other studies have also shown similar sampling variability [13]-[14].

Therefore, in clinical practice, ultrasound is often used to assess NAFLD. However, the lack of sharpness due to noise limited its role in the classification of the degree of steatosis [15]-[16].

Parficio and al. [5] classified ultrasound images were graded independently for presence and the severity of steatosis by two radiologists. Steatosis was defined by an appearance of hepatic parenchymal in which the liver was considered be normal if there was normal liver echo texture with clear visualization of the internal vascular system. The severity of steatosis was classified as mild, moderate or severe according to previously defined criteria. In fact, mild steatosis was recognized by a slight increase in the echogenicity of the liver parenchymal or no posterior beam attenuation.

Severe steatosis was recognized by coarsely increased hepatic parenchymal echotexture and subsequently marked beam attenuation. Moderate steatosis was recognized by ultrasound characteristics of liver ultrasound texture, and beam attenuation between light and severe parameters. 
$\mathrm{CT}$ is an x-ray imaging technique, Given its imprecision in detecting mild hepatic steatosis and potential radiation risk, computed tomography is not suitable for the evaluation of hepatic steatosis in the general population, but can be effective in specific clinical situations, such as assessing donor candidates for liver transplantation [17].

Disease assessment within clinical practice for NAFLD is currently done with MRI. In contrast to other imaging techniques such as ultrasound and computed tomography, which use proxies to assess hepatic steatosis (i.e., attenuation and echogenicity), Seung Soo Lee and Seong Ho Park in [17] and Parambir S.Dulai, Claude B.Sirlin, Rohit Loomba in [18] shows that magnetic resonance spectroscopy (MRS) and magnetic resonance imaging are the most accurate and reliable methods of quantifying liver fat. In our article, we are focused on magnetic resonance imaging.

Several MRI methods have been introduced to quantify hepatic fat, including chemical-shift imaging (CSI) to differentiate protons in fat from those in water, that is, the difference in MRI frequency between protons in fat and water [19]. Other methods used fat saturation, and fat-selective excitation approaches [20]-[21]. The CSI approach is most widely used because of its easy applicability and higher accuracy. Indeed, CSI techniques separate magnetic resonance (MR) signals into water (W) and fat (F) components based on the chemical shift between fat and water.

The diagnosis of fatty liver often involves the use of conventional measurement tools, But these methods remain inappropriate, therefore, other criteria must be taken into diagnostic hepatic steatosis, MRI, non- invasive examination, provide multi parametric information, a high-resolution image with an absence of completely harmless radiation.

In this paper we will propose a new approach for noninvasive quantification of intraperitoneal fat. Therefore, we wish to evaluate our study on cohort of data composed of four groups in order to prove that hepatic fat is able to differentiate patients with metabolic diseases' obesity, T2D, metabolic syndrome and healthy subjects. This distinction is according to the amount of fat from segmentation of MRI DIXON and T1mapping images at $1.5 \mathrm{~T}$ in the first heading. In the second heading, this study investigates the relationship between the correlation of hepatic fat and cardiovascular disorders. Finally, we will predict cardiovascular complications for these patients.

\section{Proposed SOlution}

To quantify the hepatic fat, we treated the water cards obtained from a specific DIXON sequence; Indeed Dixon imagery is based on the chemical displacement between water and fat protons, in order to separate their signal. In-Phase (IP): the total signal corresponds to the water signal to which is added that of the fat like Eq. (1). Out-of-Phase (OP): in Eq. (2) the total signal corresponds to the water signal from which the fat signal subtracts. It consists of making two spin echo acquisitions: the first for which the water and fat protons are in phase and the second signal for which the water and fat protons are out of phase. In Eq. (3) by adding the two signals, only that of water is displayed; by subtraction, we erase the water signal in favor of that of fat as presented in Eq. (4), results are shown in Fig. 1.

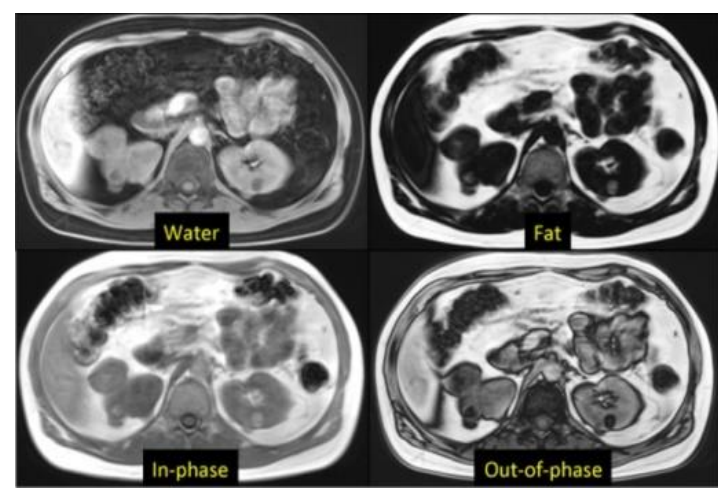

Fig. 1. Dixon Imaging.

Dixon imaging is based on the chemical displacement between water and fat protons, in order to separate their signal.

$\mathrm{IP}=\mathrm{W}+\mathrm{F}$

$\mathrm{IP}=\mathrm{W}-\mathrm{F}$

$1 / 2[\mathrm{IP}+\mathrm{OP}]=1 / 2[(\mathrm{~W}+\mathrm{F})+(\mathrm{W}-\mathrm{F})]=1 / 2[2 \mathrm{~W}]=\mathrm{W}$

$\longrightarrow$ Water only image

$1 / 2[\mathrm{IP}-\mathrm{OP}]=1 / 2[(\mathrm{~W}+\mathrm{F})-(\mathrm{W}-\mathrm{F})]=1 / 2[2 \mathrm{~F}]=\mathrm{F}$

\section{Fat only image}

\section{SOLUTION}

MRI acquisitions including DIXON imaging were performed at $1.5 \mathrm{~T}$ in 117 individuals $(60$ women, 50 men, age $47.5 \mathrm{ds}$ ): 15 obese patients, 25 metabolic syndrome patients, 40 type 2 diabetes patients and 19 healthy controls. 40 axial slices with $3 \mathrm{~mm}$ thickness, and in-plane resolution of $1.18 \mathrm{~mm}$ were acquired for each subject using a two-point Dixon sequence.

The segmentation process developed for liver fat quantification consists of three steps.

A first preprocessing step is needed to improve the quality of the MRI-DIXON-water map image. The second step is to segment the liver by combining different image processing methods (active contour Federal Trade Commission (FTC) with a double cycle of smoothing and regulation, a K-means machine learning method and mathematical morphology). The last step is to classify the liver into three classes. A class corresponds to liver fat, a class contains the vessels are presented in Fig. 2.

\section{A. Pretreatment}

To get better liver segmentation, the pretreatment step is essential before the segmentation process. In this step, we proposed the use of a morphological filter called "top hat". The principle consists in calculating the opening of the image by a very specific structuring element then to subtract the result obtained from the original image. The morphological operation 'opening' consists in eroding the image followed by dilation by the same structuring element. Using the top hat in this study makes it possible to fill in the holes and correct the intensity inconsistency in the T1 card. 

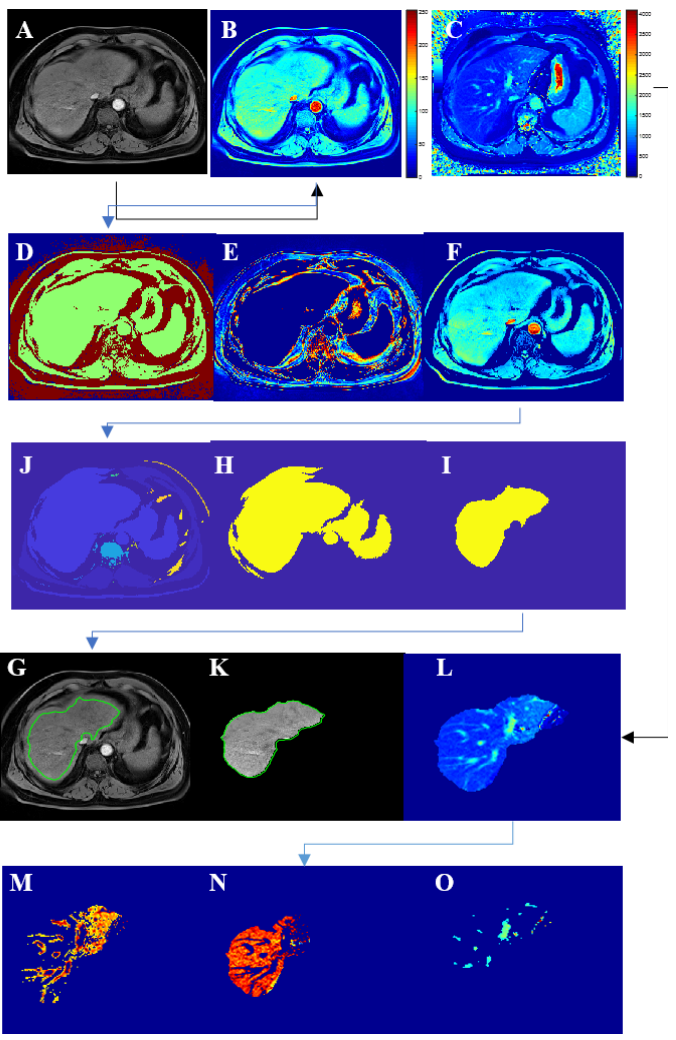

Fig. 2. Diagram of the Proposed Approach to Liver Segmentation: A: Fat Map; B: Pretreatment; C: T1-Mapping;D: Classification of the Water Map Into 2 Classes; E: Background of the Image; Liver Class; J: Distribution of the Image into Objects; H: the Largest Object Area; Mathematical Morphology Applied on (H); G: Active Contour on the Water Map; L: Superposition of the

Liver Obtained on the T1-Mapping; M: Vessel; N: Liver Fat; O: Partial Volume.

Thus, we normalize and enhance the contrast of the image by adjusting the initial histogram values. This increases the contrast of the fat in relation to the background of the image and eliminates the shadow effects linked to the acquisition artifacts and to always have the same threshold.

\section{B. Normalization}

Normalization of an image consists in dividing each value of the histogram by the total number of pixels of the image to obtain a normalized histogram. This histogram corresponds to an empirical probability distribution (all values are between 0 and 255). In Eq. (5) the formula used is as follows:

$\mathrm{I}(\mathrm{x}, \mathrm{y})=255^{*} \mathrm{I}(\mathrm{x}, \mathrm{y}) / \max (\mathrm{I}(\mathrm{x}, \mathrm{y}))$ el.

With $I$ the original image, $x$ and $y$ the coordinates of a pix-

\section{Adjustment}

Is to increase the contrast of the image, we were able to better distinguish fatty liver (in cases). The MATLAB function used is "Imadjust" with the default parameters. Thanks to this function, the image contrast is enhanced and the liver is more visible.

\section{Liver Segmentation}

This step consists in segmenting the liver from a water card obtained by the DIXON method by K-means (three classes) and choosing the liver class. To do this we distribute the image into objects. We get a card in which each object is labeled. The MATLAB function used is "bwlabel" with the default parameters (4 connections). Then the object with the largest area (the first part of the fat) as shown in Fig. 2 will be removed from the image. It is this object which corresponds to the mark of the liver.

Then, we used the mask of this object as an initialization of FTC and finally superimpose the mask obtained on the T1mapping card to have only the liver and quantify the hepatic fat. The principle of an active contour consists in positioning the image, more precisely in the vicinity of the shape to be detected, an initial contour which will undergo a deformation under the effect of several forces such as: An internal energy $E$ internal allowing to regularize the contour a potential energy $E$ image linked to the image; An external energy $E$ external linked to the particular constraints that can be added. These energies will allow the active contour to evolve to explore the minimum energy position which will thus be an arrangement between the various constraints of the problem.

\section{E. Quantification of Hepatic Fat}

For the fat quantization part, we classify the pixels of the time by the k-means algorithm into two classes.

$\mathrm{K}$-means is a data partitioning algorithm (the pixels of the image in our case). The principle is as follows: given a set of pixels in the image $(x 1, x 2, \ldots, x n)$, we seek to partition the $\mathrm{n}$ pixels into sets $S=\{S 1, S 2, \ldots, S k\}(k \leqslant n)$ by minimizing the distance between the pixels inside each partition. In this case, the number of partitions is equal to two. The formula used to quantify hepatic fat from T1-mapping and DIXON- MRI images is the average of all the pixels of the fat class.

For the evaluation of my approach, metrics will be calculated to demonstrate the strengths of my application, such as inter / intra-operator reproducibility, the Dice coefficient, the coefficient of variation (CV) and the segmentation speed.

\section{RESULT AND DISCUSSION}

Our objective in this study (in-vivo) is to propose a new approach to characterize and quantify hepatic fat and differentiate patients with metabolic diseases' obesity, T2D, metabolic syndrome and healthy subjects not only according to conventional measurement tools but also according to the amount of fat from the segmentation of DIXON MRI images and T1mapping at $1.5 \mathrm{~T}$. This first step should then make it possible to predict and assess the cardiovascular risks in these patients.

As a result, in Fig. 3, we evaluate the distribution of the quantity of hepatic fat on a cohort of data made up of four groups metabolic syndromes $(\mathrm{n}=31)$, obese $(\mathrm{n}=10)$, T2D $(\mathrm{n}=$ 48) and healthy subjects $(\mathrm{n}=19)$.

$P$ : value for quantifying the statistical significance of a result under a hypothesis 


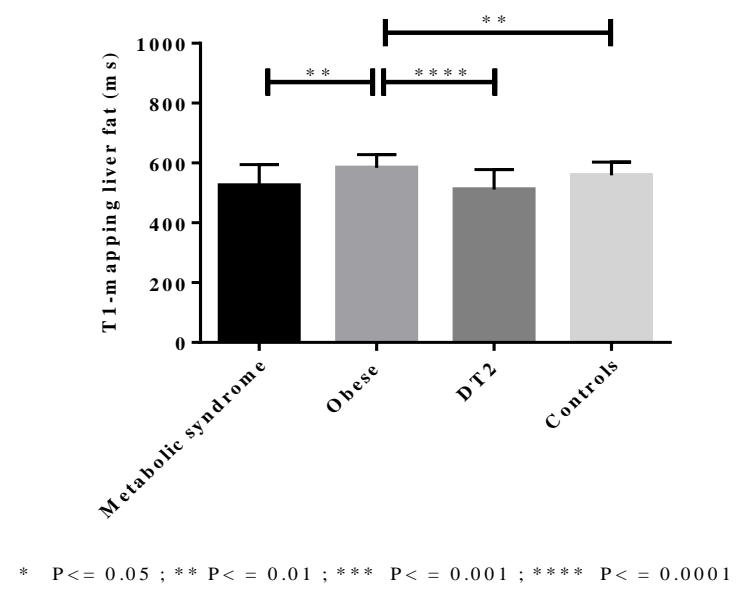

Fig. 3. Distribution of Hepatic Fat.

In the study of liver fat distribution in a population of four patient groups, significant differences were reported. The biggest difference was between obese and type 2 diabetics.

In Fig. 3, we also observe significant differences between metabolic and obese syndromes and between obese and control.

\section{A. Assessment Metric}

To assess the robustness and reproducibility of our segmentation approach, we calculated the intra-operator reproducibility by repeating the segmentation process on all groups of patients; it is defined as the absolute difference between two measurements divided by the average of two measurements. For inter-operator reproducibility, an operator who had not previously read patient data segmented the fat maps of the two groups of patients.

1) Inter-operator reproducibility: For patients with metabolic syndrome, the correlation between the fat measurements of the two operators is very good as presented in Table I. the Pearson correlation coefficient is 0.99 . The inter-operator reproducibility is $4.9 \pm 0.29 \%$. For the obese, the correlation between the fat measurements of the two operators is also very good. We obtained a Pearson correlation coefficient of 0.98 . The inter-operator reproducibility is $3.9 \pm$ $0.20 \%$. For type 2 diabetics, the correlation between the two operators' fat measurements is good. We obtained a Pearson correlation coefficient of 0.97 . The inter-operator reproducibility is $8.3 \pm 2.22 \%$. Concerning healthy subjects, the correlation between the fat measurements of the two operators is good. We obtained a Pearson correlation coefficient of 0.963 . The inter-operator reproducibility is $5.7 \pm$ 0.16 .

2) Intra-operator reproducibility: For metabolic syndromes, we obtained a Pearson correlation coefficient of 0.998 as shown in Table II. between the two measures. The intra-operator reproducibility is $3.5 \pm 1.08 \%$. Concerning T2D, we obtained a Pearson correlation coefficient of 0.973 .
3) The intra-operator reproducibility is $1.1 \pm 0.04 \%$. For the obese, the correlation between the two measures is very good. The Pearson correlation coefficient is 0.999 . The intraoperator reproducibility is $1.5 \pm 0.11 \%$. As for healthy subjects, we obtained an excellent correlation. The Pearson correlation coefficient is 0.999 . The intra-operator reproducibility is $1.6 \pm 0.075 \%$.

4) Coefficient of variation: we calculated the coefficient of variation for each group, I always obtained values lower than $14 \%$ as presented in Table III.

5) Dice index: we also calculated the Dice coefficient as shown in this Table IV.

6) Speed of the segmentation: As for the speed of segmentation, our approach is very fast in the segmentation of the liver and the quantification of hepatic fat, it allows the detection of hepatic steatosis in a time less than $5 \mathrm{~s} /$ section as presented in Table V.

TABLE. I. INTER-OPERATOR REPRODUCIBILITY: CORRELATION BETWEEN THE FAT MEASUREMENTS OF THE TWO OPERATORS ON PATIENTS WITH METABOLIC SyNDROME AND OBESE PATIENTS STYLES

\begin{tabular}{|l|l|}
\hline Groups & Pearson Correlation Coefficient \\
\hline Metabolic syndromes & 0.994 \\
\hline Obese & 0.986 \\
\hline Type 2 diabetes & 0.974 \\
\hline Control & 0.963 \\
\hline
\end{tabular}

TABLE. II. INTRA-OPERATOR REPRODUCIBILITY: CORRELATION BETWEEN THE FAT MEASUREMENTS OF THE TWO OPERATORS ON PATIENTS WITH METABOLIC SYNDROME AND OBESE PATIENTS

\begin{tabular}{|l|l|}
\hline Groups & Pearson Correlation Coefficient \\
\hline Metabolic syndromes & 0.998 \\
\hline Obese & 0.999 \\
\hline Type 2 diabetes & 0.973 \\
\hline Control & 0.999 \\
\hline
\end{tabular}

TABLE. III. COEFFICIENT OF VARIATION FOR PATIENTS WITH METABOLIC SyndROME, OBESE, T2 AND HEALThy SubJECTS

\begin{tabular}{|l|l|}
\hline Groups & $\mathbf{C V}(\boldsymbol{\%})$ \\
\hline Metabolic syndromes & 13.388 \\
\hline Obese & 7.512 \\
\hline Type 2 diabetes & 13.006 \\
\hline Control & 7.905 \\
\hline
\end{tabular}

TABLE. IV. DICE INDEX \%

\begin{tabular}{|l|l|}
\hline Groups & Liver Fat $\%$ \\
\hline Metabolic syndromes & 0.988 \\
\hline Obese & 0.974 \\
\hline Type 2 diabetes & 0.969 \\
\hline Control & 0.985 \\
\hline
\end{tabular}


TABLE. V. SPEED OF SEGMENTATION OF OUR APPROACH: THE METHOD IS VERY FAST

\begin{tabular}{|l|l|l|l|l|}
\hline \multicolumn{3}{|l|}{ Segmentation speed (s/slice) } & \\
\hline Groups & $\begin{array}{l}\text { Liver from } \\
\text { Dixon }\end{array}$ & $\begin{array}{l}\text { Liver from T1- } \\
\text { mapping (s) }\end{array}$ & $\begin{array}{l}\text { Liver } \\
\text { Quantification } \\
(\mathrm{s})\end{array}$ & Total time \\
\hline $\begin{array}{l}\text { Metabolic } \\
\text { syndrome }\end{array}$ & 2.45 & 1,87 & 0.780 & 5.10 \\
\hline Obese & 2.53 & 1,66 & 0.877 & 5.06 \\
\hline $\begin{array}{l}\text { Type 2 } \\
\text { diabetes }\end{array}$ & 2.35 & 1,87 & 0.890 & 5.11 \\
\hline Controls & 2.7 & 1,98 & 1.020 & 5.70 \\
\hline
\end{tabular}

\section{B. Statistical Study}

In this part, risk factors will be correlated with liver fat to study the metabolic links that may exist.

1) Relationship between liver fat and age: In the statistical study, we observed a significant relationship between the amount of fat and age. The amount of fat increases significantly with age for patients with metabolic syndrome $(P=0.04)$ and obese patients $(P=0.045)$ and also type 2 diabetics $(P=0.033)$.

As for the holy subject this quantity has no significant connection with age $(P=0.54)$ as shown in Fig. 4.

2) Relationship between liver fat and BMI: We also studied the correlation between the amount of fat and the body mass index. Indeed, the amount of fat tends to increase with BMI but not significantly for all groups, namely patients with metabolic syndrome $(P=0.28)$, obese $(P=0.55)$, type 2 diabetics $(P=0.08)$ and healthy subjects $(P=0.89)$ as shown in Fig. 5.

3) Relationship between liver fat and BSP: The amount of fat increases significantly with systolic brachial pressure for patients with metabolic syndrome $(P=0.049)$. However, it did not significantly for type 2 diabetics $(P=0.9)$ as shown in Fig. 6.
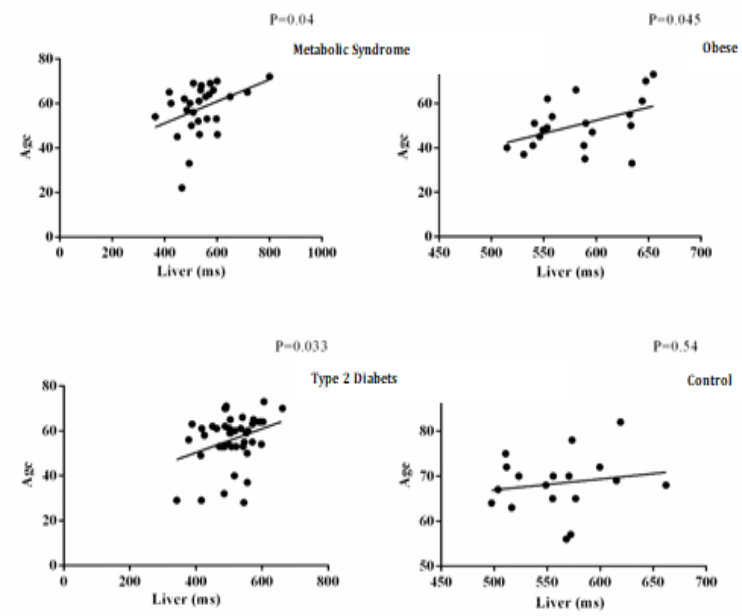

Fig. 4. Change in the Amount of Liver Fat as a Function of Age.
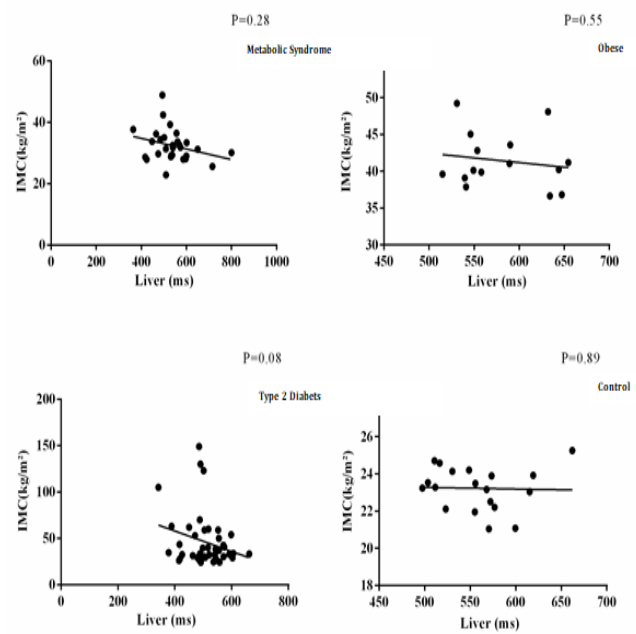

Fig. 5. Liver Fat and BMI.
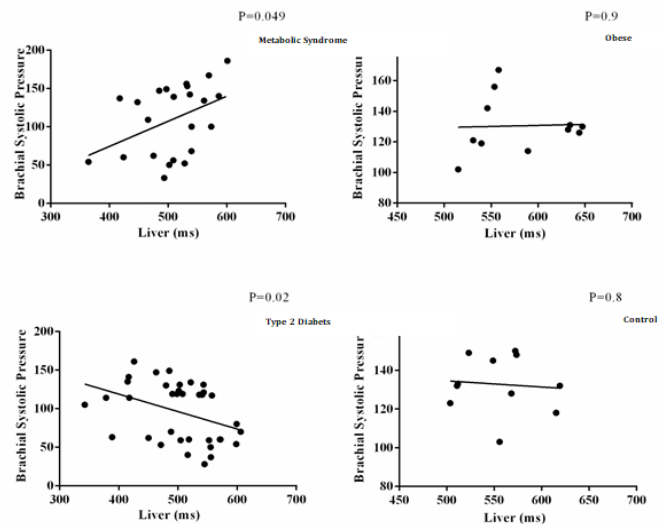

Fig. 6. Liver Fat and Brachial Systolic Pressure.

4) Relationship between liver fat and BDP: The amount of fat increases significantly with systolic brachial pressure for patients with metabolic syndrome $(P=0.01)$. However, it significantly reduced for type 2 diabetics $(P=0.04)$ as you can see in Fig. 7.
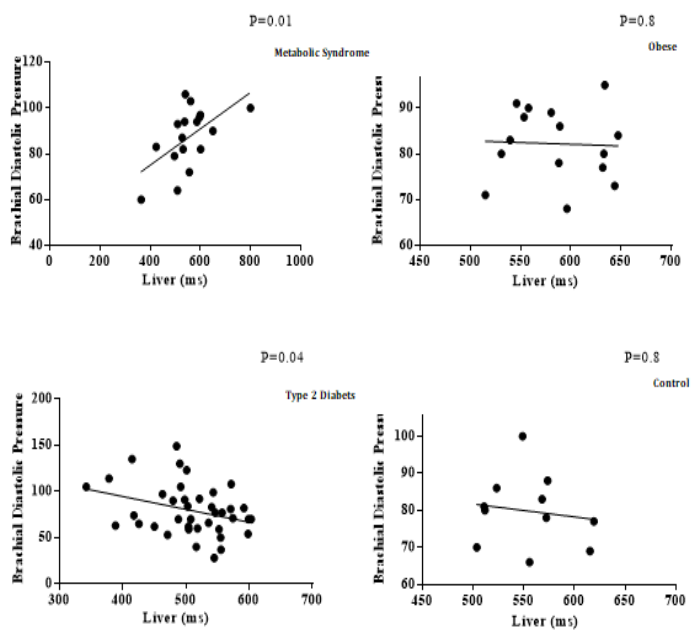

Fig. 7. Liver Fat and Brachial Dystolic Pressure. 


\section{CONCLUSION}

In conclusion, the precise classification and quantification of hepatic fat is crucial for metabolic studies and the detection of fibrosis where they serve as good indicators of the associated metabolic and cardiovascular disorders. They can serve as an effective and precise tool for the diagnosis and differentiation of risk profiles of patients with metabolic diseases and could be considered in the future to predict cardiovascular complications. Relationship study of hepatic fat and cardiovascular parameters shows that hepatic fat has a negative influence on the heart if the amount it increases.

The perspectives of this work are many; first we want to segment the liver by deep learning algorithms. Then we also want to detect and quantify hepatic fibrosis from the T1 mapping.

\section{REFERENCES}

[1] Tsiplakidou, M., Tsipouras, M. G., Manousou, P., Giannakeas, N., \& Tzallas, "Automated Hepatic Steatosis Assessment through Liver Biopsy Image Processing", IEEE 18th Conference on Business Informatics (CBI), 2016; DOI:10.1109/cbi.2016.51.

[2] Z.M. Younossi, Deirdre Blissett, Robert Blissett, Linda Henry, Maria Stepanova, Andrei Racila, "The economic and clinical burden of nonalcoholic fatty liver disease in the United States and Europe", Hepatology, 2016; vol. 64, no. 5, pp. 1577-1586.

[3] Loomba R, Sanyal AJ, "The global NAFLD epidemic", Nat Rev Gastroenterol Hepatol, 2013;10:686-690.

[4] Rinella ME, "Nonalcoholic fatty liver disease: a systematic review", JAMA, 2015;313:2263-2273.

[5] Lucia Pacifico, Michele Di Martino, Carlo Catalano, Valeria Panebianco, Mario Bezzi, Caterina Anania, and Claudio Chiesa, "T1weighted dual-echo MRI for fat quantification in pediatric nonalcoholic fatty liver disease", World Journal of Gastroenterology: WJG, 2011;17: 30129.

[6] Spengler EK, Loomba R, "Recommendations for diagnosis, referral for liver biopsy, and treatment of nonalcoholic fatty liver disease and nonalcoholic steatohepatitis", Mayo Clin Proc, 2015;90:1233-1246.

[7] Chalasani N, Younossi Z, Lavine JE, Diehl AM, Brunt EM, Cusi K, Charlton M, Sanyal AJ, "The diagnosis and management of nonalcoholic fatty liver disease: practice guideline by the American Gastroenterological Association, American Association for the Study of Liver Diseases, and American College of Gastroenterology", Gastroenterology,2012;142:1592-1609.

[8] Arulanandan A, Ang B, Bettencourt R, Hooker J, Behling C, Lin GY, Loomba R.,"Association between quantity of liver fat and cardiovascular risk in patients with nonalcoholic fatty liver disease independent of nonalcoholic steatohepatitis", Clin Gastroenterol Hepatol, 2015;13:1513-1520.

[9] A. Han, J. W. Erdman, D. G. Simpson, M. P. Andre and W. D. O'Brien, "Early detection of fatty liver disease in mice via quantitative ultrasound", IEEE International Ultrasonics Symposium, Chicago, IL, 2014; pp. 2363-2366.

[10] J. Kong, J. Lee Michael, P. Bagci, P. Sharma, D. Martin, N. Volkan Adsay, J. H. Saltz and A. B. Farris, "Computer-based Image Analysis of Liver Steatosis with Large-scale Microscopy Imagery and Correlation with Magnetic Resonance Imaging Lipid Analysis", IEEE [International Conference on Bioinformatics and Biomedicine], 2011; 978-0-76954574-5/11.

[11] Bonekamp S, Tang A, Mashhood A, S Michael S. Middleton MD , "Spatial distribution of MRI Determined hepatic proton density fat fraction in adults with nonalcoholic fatty liver disease", J Magn Reson Imaging, 2014;39:1525-1532.

[12] Diana Feier, Ahmed Ba-Ssalamah, "Current Noninvasive MR-Based Imaging Methods in Assessing NAFLD Patients", IntechOpen, May 31st 2019; DOI: 10.5772/intechopen.82096.

[13] T. Nguyen, A. Podkowa, R. J. Miller, M. L. Oelze and M. Do, "In-vivo study of quantitative ultrasound parameters in fatty rabbit livers", IEEE International Ultrasonics Symposium (IUS), Washington, DC, 2017; pp. $1-4$.

[14] Harald Kramer, Perry J. Pickhardt, Mark A. Kliewer, Diego Hernando, Guang-Hong, "Accuracy of liver fat quantification with advanced CT MRI and Ultrasound techniques: prospective comparison with MR spectroscopy”, AJR Am. J Roentgenol, 2017; vol. 208, no. 1, pp. $92-$ 100, Jan.

[15] Aiguo Han ; Andrew S. Boehringer ; Yingzhen N. Zhang ; Vivian Montes ; Michael P. Andre, "Improved Assessment of Hepatic Steatosis in Humans Using Multi-Parametric Quantitative Ultrasound", IEEE International Ultrasonics Symposium (IUS), Glasgow, United Kingdom, 2019; pp. 1819-1822.

[16] Che-Chou Shen ; Sheng-Chang Yu ; Chia-Yuan Liu, "Using highfrequency ultrasound statistical scattering model to assess Nonalcoholic Fatty Liver Disease (NAFLD) in mice", International Conference on Telecommunications and Signal Processing (TSP), Vienna, 2016; pp. 379-382.

[17] Seung Soo Lee and Seong Ho Park, "Radiologic evaluation of nonalcoholic fatty liver diseas", World J Gastroenterol, 2014 Jun 21; 20(23): 7392-7402.

[18] Parambir S.Dulai, Claude B.Sirlin, Rohit Loomba, "MRI and MRE for non-invasive quantitative assessment of hepatic steatosis and fibrosis in NAFLD and NASH: Clinical trials to clinical practice", Journal of hepatology, 2016; vol. 65: 1006-1016.

[19] Reeder SB, Cruite I, Hamilton G, Sirlin CB, "Quantitative Assessment of Liver Fat with Magnetic Resonance Imaging and Spectroscopy", J Magn Reson Imaging, 2011 Oct; 34(4):729-749.

[20] J. Cui, B. Ang, W. Haufe, C. Hernandez, E. C. Verna, C. B. Sirlin, R. Loomba, "Comparative diagnostic accuracy of magnetic resonance elastography vs. eight clinical prediction rules for non-invasive diagnosis of advanced fibrosis in biopsy-proven non-alcoholic fatty liver disease: a prospective study", Aliment Pharmacol Ther, 2015;41: 12711280.

[21] Rohit Loomba, Tanya Wolfson, Brandon Ang, Jonathan Hooker, Cynthia Behling, Michael Peterson, "Magnetic resonance elastography predicts advanced fibrosis in patients with nonalcoholic fatty liver disease: a prospective study", Hepatology, 2014;60:1920-1928. 